\title{
An Analytic Soluion of Fingering Phoenomenon Arising in Fluid Flow through Porous Media by Using Techniques of Calculus of Variation and Similarity Theory
}

\author{
Bani Mukherjee (Corresponding Author) \& Pinki Shome \\ Department of Applied Mathematics \\ Indian School of Mines University \\ Dhanbad 826004, Jharkhand, India
}

Tel: 91-943-112-5407Ｅ-mail: mukherjeebani@gmail.com

\begin{abstract}
The present paper represents an analytical solution of fingering phonomenon arising in double phase flow through homogeneous media under certain initial \& boundary condition using techniques of calculus of variation and similarity theory. The numerical and graphical representation of solution has been given the graph of saturatin $F(\eta)$ of injected liquid, is increasing after $\eta=0.5$ for $t>0$, which indicates that when injected liquid entries into native liquid at common-interface, then suddenly the native liquid enters into injected liquid due to difference in wettability. Hence initial saturation will decrease and then after $\eta>0.5$ the saturation uniformly increases parabolically which is physically consistent with the available theory.
\end{abstract}

Keywords: Fingering phenomenon, Double phase flow, Similarity theory, Capillary force, Calculus of Variation, RayleighRitz method

$\begin{array}{ll}\text { Nomenclature } \\ V_{W} & \text { Seepage velocity of Water } \\ V_{0} & \text { Seepage velocity of Oil } \\ \rho_{W} & \text { Density of Water } \\ \rho_{0} & \text { Density of Oil } \\ \alpha & \text { The inclinati of the bed, } \\ g & \text { Acceleratin due to gravity, } \\ P_{c} & \text { Capillary pressure } \\ P_{0} & \text { Pressure of Oil } \\ P_{W} & \text { Pressure of Water } \\ P & \text { Mean Pressure } \\ S_{W} & \text { Saturation of Water } \\ S_{0} & \text { Saturation of Oil } \\ \eta(x, t) & \text { The interface curve } \\ K & \text { Intrinsic permeability }\end{array}$

\section{Introduction}

It is a very well-known physical fact that when a fluid, contained in a porous media, is displaced by another of lesser viscosity, instead of regular displacement of whole front, perturbations (fingers) occur which shoot through the porous medium at relatively great speed. This phenomenon of occurrence of instabilities is called fingering.

Immiscible flow of heavy oil in a porous formation by high temperature pressurized water has been numerically studied. The physical region is a square domain in the horizontal plane with low and high pressure points at the opposite corners along one of the diagonals. Water, the invading fluid, when introduced at high pressure displaces the in situ oil towards the low-pressure production zone. The extent of displacement of oil by water through the porous medium in a given amount of time and the appearance of preferential flow paths (fingers) is the subject of the present investigation. 
The resistance to water-oil movement arises from the viscous forces in the fluid phases and the capillary force at their interface. Based on their relative magnitudes, various forms of displacement mechanisms can be realized. As the viscosity ratio of heavy oil to water is large, viscous forces in the oil phase become dominant and constitute the major factor for controlling the flow distortions in the porous formation. A mathematical model that can treat the individual fluid pressures, capillary effects and heat transfer has been employed in the present work. A fully implicit, two-dimensional numerical model has been used to compute the pressure and temperature fields. The domain decomposition technique has been adopted in the numerical solution since the problem is computationally intensive. Naturally occurring oil-rich reservoirs to which the present study is applicable are inhomogeneous and layered. A qualitative study has been carried out to explore the effect of permeability variations on the flow patterns. Numerical calculations show that non-isothermal effects as well as layering, promote the formation of viscous fingers and consequently the sweep efficiency of the high-pressure waterfront.

In the statistical treatment of fingering (Scheidegger, A.E., 1961) only average cross sectional area occupied by the fingers, is taken into account, the size and shape of the individual fingers are disregarded. Scheideger and Johnson (1961) introduce the idea of discussing the statistical behaviour of instabilities in homogeneous porous media and considered the phenomenon without the effecct of capillary pressure. Verma (1964) has examined the behaviour of fingering phenomenon in a displacement process through heterogeneous porous medium from statistical point of view. It has been shown that fingers may be stabilized in homogeneous media statistical view point by (Scheidegger, A.E., 1960) many authors, for example, Chouke (1959), Jecquard (1940), Verma (1924), have investigated this phenomenon with different aspects.

\subsection{Formulation of Problem}

Let water be injected with constant velocity into a dipping oil saturated porous medium of homogeneous physical characteristic. The displacement of oil by water gives rise to a well-developed finger flow as shown in Figure 1(a), 1(b).

$<$ Figure 1(a) \& Figure 1(b) >

From Darcy's law the seepage velocity of water $V_{w}$ and oil $V_{0}$ can be written as

$$
\begin{aligned}
& V_{w}=\frac{K_{w}}{\delta_{w}} K\left(\frac{\partial P_{w}}{\partial X}+\rho_{w} g \sin \alpha\right) \\
& V_{0}=-\frac{K_{0}}{\delta_{0}} K\left(\frac{\partial P_{0}}{\partial X}+\rho_{0} g \sin \alpha\right)
\end{aligned}
$$

where $\rho_{w}$ and $\rho_{0}$ are constant densities of water and oil respectively, $\alpha$ is the inclination of the bed, $g$ is acceleration due to gravity.

The equation of continuity of the phases is given by

$$
\begin{aligned}
& P \frac{\delta S_{w}}{\partial_{t}}+\frac{\partial V_{w}}{\partial X}=0 \\
& P \frac{\delta S_{0}}{\partial_{t}}+\frac{\partial V_{0}}{\partial X}=0
\end{aligned}
$$

From the definition of phase saturation we have

$$
S_{w}+S_{0}=1
$$

The capillary pressure, which is defined as the pressure discontinuity of the following phases across the common interface is written as

$$
P_{c}=P_{0}-P_{w}
$$

Or

$$
\frac{\partial P_{w}}{\partial X}=\frac{\partial P_{0}}{\partial X}-\frac{\partial P_{c}}{\partial X}
$$

The equation of motion for saturation can be obtained by substitution of the values of $V_{w}$ and $V_{0}$ from equations (1) and (2) in equation (3) and (4) respectively. Thus we have

$$
\begin{gathered}
P \frac{\partial S_{w}}{\partial t}=\frac{\partial}{\partial X}\left[\left(\frac{K_{w}}{\delta_{w}} k\right)\left(\frac{\partial P_{w}}{\partial X}+\rho_{w} g \sin \alpha\right)\right] \\
P \frac{\partial S_{0}}{\partial t}=\frac{\partial}{\partial X}\left[\left(\frac{K_{0}}{\delta_{0}} k\right)\left(\frac{\partial P_{0}}{\partial X}+\rho_{0} g \sin \alpha\right)\right]
\end{gathered}
$$

Substituting the value of $\frac{\partial P_{w}}{\partial X}$ from Eq. (7), Eq. (8) reduces to

$$
P \frac{\partial S_{w}}{\partial t}=\frac{\partial}{\partial X}\left[\left(\frac{K_{w}}{\delta_{0}} k\right)\left(\frac{\partial P_{0}}{\partial X}-\frac{\partial P_{c}}{\partial X}+\rho_{w} g \sin \alpha\right)\right]
$$


Now considering Eq. (9) and (10)

$$
P \frac{\partial}{\partial t}\left(S_{w}+S_{0}\right)=\frac{\partial}{\partial X}\left[\frac{\partial P_{0}}{\partial X} k\left(\frac{K_{0}}{\delta_{0}}+\frac{K_{w}}{\delta_{w}}\right)-\frac{K_{w}}{\delta_{w}} K \frac{\partial P_{0}}{\partial X}+g \sin \alpha K\left(\frac{K_{0} \rho_{0}}{\delta_{0}}+\frac{K_{w} \rho_{w}}{\delta_{w}}\right)\right]
$$

Using (5) we have

$$
\frac{\partial}{\partial X}\left[\frac{\partial P_{0}}{\partial X} K\left(\frac{K_{0}}{\delta_{0}}+\frac{K_{w}}{\delta_{w}}\right)-K \frac{K_{w}}{\delta_{w}} K \frac{\delta P_{0}}{\delta X}+K g \sin \alpha\left(\frac{K_{0} \rho_{0}}{\delta_{0}}+\frac{K_{w} \rho_{w}}{\delta_{w}}\right)\right]=0
$$

Integrating both sides w.r.t. " $x$ " we have

$$
K \frac{\partial P_{0}}{\partial X}\left(\frac{K_{0}}{\delta_{0}}+\frac{K_{w}}{\delta_{w}}\right)-K \frac{K_{w}}{\delta_{w}} \frac{\partial P_{0}}{\partial X}+K g \sin \alpha\left(\frac{K_{0} \rho_{0}}{\delta_{0}}+\frac{K_{w} \rho_{w}}{\delta_{w}}\right)=\text { cons } \tan t
$$

or,

$$
\left(\frac{K_{w}}{\delta_{w}}+\frac{K_{0}}{\delta_{0}}\right) \frac{\partial P_{0}}{\partial X} K-\frac{K_{w}}{\delta_{w}} K+g K \sin \alpha\left(\frac{K_{0} \rho_{0}}{\delta_{0}}+\frac{K_{w} \rho_{w}}{\delta_{w}}\right)=-q
$$

where $q$ is the constant of integration

or,

$$
\frac{\partial P_{0}}{\partial X}=\frac{\left[-q+\frac{K_{w}}{\delta_{w}} \frac{\partial P_{0}}{\partial X}-g K \sin \alpha\left(\frac{K_{0} \rho_{0}}{\delta_{0}}+\frac{K_{W} \rho_{W}}{\delta_{W}}\right)\right]}{\left(\frac{K_{W} K}{\delta_{W}}+\frac{K_{0} K}{\delta_{0}}\right)}
$$

Substituting the value of $\frac{\partial P_{0}}{\partial X}$ from equation (13), equation (10) becomes (Appendix A).

$$
P \frac{\partial S_{W}}{\partial t}+\frac{\partial}{\partial X}\left[\frac{\frac{K_{0} K}{\delta_{0}}\left\{\frac{\partial P_{0}}{\partial X}+g \sin \alpha\left(\rho_{0}-\rho_{w}\right)\right\}}{\left(1+\frac{K_{0} \delta_{W}}{K_{w} \delta_{0}}\right)}+\frac{q}{\left(1+\frac{K_{0} \delta_{W}}{K_{w} \delta_{0}}\right)}\right]=0
$$

The value of the pressure of oil $\left(P_{0}\right)$ can be written as

$$
P_{0}=\left(P_{0}+P_{W}+P_{0}-P_{W}\right) / 2=\frac{P_{0}+P_{W}}{2}+\frac{P_{0}-P_{W}}{2}=\bar{P}+\frac{1}{2}\left(P_{0}-P_{W}\right)=\bar{P}+\frac{1}{2} P_{c}
$$

Where $\bar{P}$ is the mean pressure. Since $\bar{P}$, the mean pressure is constant hence we have

$$
\frac{\partial P_{0}}{\partial X}=\frac{1}{2} \frac{\partial P_{c}}{\partial X}
$$

Substituting the value of $\frac{\partial P_{0}}{\partial X}$ in equation (12) we get (Appendix B)

$$
q=\frac{\partial P_{c}}{\partial X}\left(\frac{1}{2} \frac{K_{W} K}{\delta_{W}}-\frac{1}{2} \frac{K_{0} K}{\delta_{0}}\right)-g \sin \alpha\left(\frac{K_{0} K \rho_{0}}{\delta_{0}}+\frac{K_{W} K \rho_{W}}{\delta_{W}}\right)
$$

using the above value of " $q$ " equation (14) reduces to (Appendix C)

$$
P \frac{\partial S_{W}}{\partial t}+\frac{\partial}{\partial X}\left[\frac{K_{w} K}{\delta_{w}}\left\{\frac{1}{2} \frac{\partial P_{0}}{\partial X}-\rho_{w} g \sin \alpha\right\}\right]=0
$$

\subsection{A Special case study}

For definitions of the mathematical analysis, we assume a standard form for the relationship between capillary pressure, permeability of water \& permeability of oil with phase saturation as

$$
\begin{gathered}
K_{w}=S_{w} \\
K_{0}=1-S_{w} \\
P_{0}=B\left(S_{w}^{-\frac{1}{2}}-C\right)
\end{gathered}
$$

where $B$ and $C$ are constant.

Using the above values equation (18) reduces as follow :

$$
P \frac{\partial S_{w}}{\partial t}+\frac{\partial}{\partial X}\left[\frac{S_{w} K}{\delta_{W}}\left\{\frac{-B}{4} S_{w}^{-\frac{3}{2}} \frac{\partial S_{w}}{\partial X}+\rho_{w} g \sin \alpha\right\}\right]=0
$$


This is the equation of motion for saturation, with the boundary condition

$$
\text { when } x=0 \text { then } S_{w}(0, t)=S_{w 0},
$$

Since an exact solution of equation (20) is difficult to obtain due to non-linear forms there in hence we have obtain the approximate solution of the above problem by Rayleigh-Ritz method :

\subsection{Solution procedure}

Since capillary pressure is very small in porous media, hence assuming that the capillary pressure $P_{c}$ as zero or $B$ as zero, equation (20) reduces to

$$
P \frac{\partial S_{w}}{\partial t}+\left[\frac{\rho_{w} g \sin \alpha}{\delta_{w}} \frac{\partial S_{w}}{\partial X} K\right]=0
$$

or,

$$
\frac{\partial S_{w}}{\partial t}+\delta \frac{\partial S_{w}}{\partial X}=0
$$

where

$$
\delta=\rho_{w} g \sin \alpha\left(\frac{K}{P \delta_{w}}\right)
$$

Using Birkhof's technique of one parameter group transformation, defined as

$$
\begin{gathered}
T_{1}: X=a^{q} x \\
T=a^{u} t \\
\overline{S_{w}}=a^{v} S_{w}
\end{gathered}
$$

where parameter $a \neq 0$, and $q, u, v$ are real numbers to be determined.

Hence we have

$$
\begin{aligned}
& \frac{\partial S_{w}}{\partial t}=a^{-v} \frac{\partial \overline{S_{w}}}{\partial T} \frac{\partial T}{\partial t}=a^{u-v} \frac{\partial \overline{S_{w}}}{\partial T} \\
& \frac{\partial S_{w}}{\partial X}=a^{-v} \frac{\partial \overline{S_{w}}}{\partial X} \frac{\partial X}{\partial x}=a^{q-v} \frac{\partial \overline{S_{w}}}{\partial X}
\end{aligned}
$$

Equation (21) using above value becomes

$$
a^{u-v} \frac{\partial \overline{S_{w}}}{\partial T}+\delta a^{q-v} \frac{\partial \overline{S_{w}}}{\partial X}=0
$$

equation (24) is absolute conformed invaraint under $T_{1}$ provided

$$
q-v=u-v
$$

or,

or,

$$
\frac{q}{u}-\frac{v}{u}=1-\frac{v}{u}
$$

$$
\frac{q}{u}=1
$$

and choosing an arbitrary constant " $A$ " as follows

$$
\frac{v}{u}=A
$$

Thus the invariants of group $T_{1}$ is given by

$$
\eta=\frac{x}{l}
$$

and

or,

$$
\begin{gathered}
F(\eta)=\frac{S_{w}(x, t)}{t^{A}} \\
S_{w}(x, t)=t^{A} F(\eta) \\
S_{w}=t^{A} F \\
\frac{\partial S_{w}}{\partial t}=A t^{A-1} F+t^{A} F^{\prime}\left(-\frac{x}{t^{2}}\right),
\end{gathered}
$$


where dash represent differentiations w.r.t. ' $\eta$ '

$$
\frac{\partial S_{w}}{\partial t}=t^{A-1}\left(A F-\eta F^{\prime}\right)
$$

and

$$
\frac{\partial S_{w}}{\partial x}=t^{A-1} F^{\prime}(\eta)
$$

Substituting the above values in equation (21) we have

$$
t^{A-1}\left[A F(\eta)-\eta F^{\prime}(\eta)+\delta F^{\prime}(\eta)\right] 0
$$

Since $t^{A-1} \neq 0$, therefore

$$
A F(\eta)-\eta F^{\prime}(\eta)+\delta F^{\prime}(\eta)=0
$$

This is an ordinary differential equation of first order.

\section{Case 1.}

Let trial solutionis :

$$
F(\eta)=a_{1} a_{2} \eta
$$

since

$$
\begin{gathered}
\eta \rightarrow 0, F \rightarrow F_{0} \\
F(0.1)=a_{1}=F_{a}
\end{gathered}
$$

or,

$$
F=F_{0}+a_{2} \eta
$$

hence or,

$$
F^{\prime}=a_{2}
$$

substituting the value of $F, F^{\prime}$ we have

$$
A\left(F_{0}+a_{2} \eta\right)-\eta a_{2}+\delta a_{2}=0
$$

or,

$$
\left(A F_{a}+\delta a_{2}\right)+\eta\left(A a_{2}-a_{2}\right)=0
$$

equating the coefficient of $\eta$ both sides

$$
\begin{gathered}
A=1 \\
a_{2}=\frac{-A F_{0}}{\delta}=\frac{-F_{0}}{\delta} \\
F=\left(F_{0}-\frac{F_{0}}{\delta} \eta\right)=F_{0}\left(1-\frac{\eta}{\delta}\right)=F_{0}\left(1-\frac{x}{\delta . t}\right)
\end{gathered}
$$

Hence from equation (29) that is $S_{w}=t^{2} F(\eta)$ we have

$$
S_{w}=t^{2} F(\eta)=F_{0}\left(1-\frac{x}{\delta . t}\right) t^{2}=\frac{S_{w 0}}{t^{A}}\left(1-\frac{x}{\delta t}\right) t^{2}=\frac{S_{w 0}}{t^{A-1}}\left(t-\frac{x}{\delta}\right)
$$

\section{Case 2.}

Now we solve the above equation (30) or $A F(\eta)-\eta F^{\prime}(\eta)+\delta F^{\prime}(\eta)=0$ with the boundary condition (23) that is when $x=0$ then $S_{w}(0 . t)=S_{w 0}$ or in other words, when $\eta=0$, then $F(0, t)=\frac{S_{w 0}}{t^{A}}=F_{0}$ by Rayleigh-Ritz method as follows :

(1) Writing the given differential equation as the Euler's equation of some variational problem.

(2)Reducing this variational problem to a minimizing problem by assuming an approximate solution in the form

$$
\bar{y}(x)=y_{0}(x)+\sum c, \phi_{i}(x)
$$

Where the trial functions $\phi_{i}(x)$ satisfy the boundary conditions and $\phi_{i}(x)=0$ on the $C$ of its region $R$.

Let the integral to be extremised be

$$
I=\int_{a}^{b} f\left(y, y^{\prime}, x\right) d x
$$


Such that $y(a)=A$, and $y(b)=B$.

Substituting (31) in (32) by replacing $y$ by $\bar{y}$ in $I$, giving $\bar{I}$ as a function of the unknown's $c_{i}$. Then $c$ 's become parameters, which are so determiend as to extremise $\bar{I}$. This requires

$$
\frac{\partial \bar{I}}{\partial c_{i}}=0, \quad i=1,2,3,4 \ldots
$$

Solving these equations, we get the values of $c_{i}$, which when substituted in (31) give the desired solution. It's solution is equivalent to extremising the following integral :

$$
I=\int_{0}^{\delta} \phi\left(\eta, F, F^{\prime}\right) d \eta
$$

The functional of the above problem is as follows :

$$
\phi\left(\eta, F, F^{\prime}\right)=F^{2}\left(\frac{A+1}{2}\right)+(\delta-\eta) F F^{\prime}
$$

Since the Euler's equation

$$
\frac{\partial \phi}{\partial F}-\frac{d}{d x}\left(\frac{\partial \phi}{\partial F^{\prime}}\right)=0
$$

gives Eq. (35).

Now assuming the trail function as

$$
\bar{F}=F_{0}\left(1-\frac{\eta}{\delta}\right)^{a}
$$

Differentiating with respect to $\eta$ we have

$$
\bar{F}^{\prime} F_{0} \alpha\left(1-\frac{\eta}{\delta}\right)^{\alpha-1}\left(\frac{-1}{\delta}\right)
$$

Now replacing $F$ by $\bar{F}$, and substituting the values of $\bar{F}$ and $\bar{F}^{\prime}$ in equation (34) we have

or,

$$
\begin{aligned}
& \int_{0}^{\delta} \phi d \eta= \int_{0}^{\delta}\left\{(A+1) F_{0}^{2}\left(1-\frac{\eta}{\delta}\right)^{2 \alpha}\right. \\
&\left.+\delta\left(1-\frac{\eta}{\delta}\right) F_{0}\left(1-\frac{\eta}{\delta}\right)^{\alpha} F_{0} \alpha\left(1-\frac{\eta}{\delta}\right)^{\alpha-1}\left(\frac{-1}{\delta}\right)\right\} d \eta \\
&= \int_{0}^{\delta} F_{0}^{2}\left(1-\frac{\eta}{\delta}\right)^{2 \alpha}[(A+1)-\alpha] d \eta \\
&= F_{0}^{2}[(A+1)-\alpha](-\delta)\left[\frac{\left(1-\frac{\eta}{\delta}\right)}{\alpha+1}\right]^{\delta} \\
& I=\frac{F_{0}^{2} \delta}{\alpha+1}(A+1-\alpha)
\end{aligned}
$$

$$
F_{0}^{2} \delta(A+1-\alpha)=I(\alpha+1)
$$

or,

$$
\begin{gathered}
-F_{0}^{2} \delta=\frac{d l}{d \alpha}(\alpha+1)+I \\
I=\frac{F_{0}^{2} \delta}{\beta+1}(A+1-\beta)
\end{gathered}
$$

It's stationary value is given by $\frac{d I}{d \beta}=0$.

Differentiating equation (38) with respect to $\beta$ we have

$$
F_{0}^{2} \delta=\frac{d I}{d \beta}(\beta+1)+I
$$

or

$$
\frac{d I}{d \beta}=\frac{F^{2} \delta-I}{\beta+1}=0
$$




$$
\frac{F_{0}^{2} \delta}{\beta+1}(A+1-\beta)=F_{0}^{2} \delta
$$

or,

$$
A+1-\beta=\beta+1
$$

or,

$$
A=\beta
$$

Since the trail solutin of the second order functional equation will be of degree at most two hence $\beta=2$. So the approximate solution is

$$
\bar{F}=F_{0}\left(1-\frac{\eta}{\delta}\right)^{2}
$$

Hence from equation (29) that is $S_{w}=t^{2} F(\eta)$ we have

$$
S_{w}(x, t)=F_{0}\left(t-\frac{x}{\delta}\right)^{2}
$$

\section{Conclusion}

The equation

$$
S_{w}(x, t)=F_{0}\left(t-\frac{x}{\delta}\right)^{2}
$$

represents analytical solution of fingering phenomenon arising double phase flow through homogeneous media under initial \& boundary condition.

The graphical presentation has been given by figure - 3 that is the graph of $F(\eta)$ verses $\eta$. The graph of saturation $F(\eta)$ of injeted liquid is increasing after $\eta=0.5$ for $t>0$, which indicates that when injected liquid entries into native liquid at common-interface, then suddenly the native liquid enters into injected liquid due to difference in wettability.

Hence initial saturation will decrease and then after $\eta>0.5$ the saturation uniformly increases parabolically which is physically consistent with the available theory.

$<$ Table $1>$

$<$ Figure 2>

\section{APPENDIX (A)}

$$
\begin{gathered}
\frac{\partial S_{w}}{\partial t} \frac{\partial}{\partial X}\left[\frac{K_{w} K}{\delta_{w}}\left(\frac{\partial P_{0}}{\partial X}-\frac{\partial P_{0}}{\partial X}+\rho g \sin \alpha\right)\right] \\
\frac{\partial P_{0}}{\partial X}=\frac{-q+\frac{K_{w} K}{\delta_{w}} \frac{\partial P_{0}}{\partial X}-g K \sin \alpha\left(\frac{K_{0} \rho_{0}}{\delta_{0}}+\frac{K_{w} \rho_{w}}{\delta_{W}}\right)}{\left(\frac{K_{W} K}{\delta_{W}}+\frac{K_{0} K}{\delta_{0}}\right)}
\end{gathered}
$$

Substituting the value of (A.2), (A.1) becomes

$$
\begin{aligned}
& \frac{\partial S_{w}}{\partial t}=\frac{\partial}{\partial X}\left[\frac{K_{w} K}{\delta_{w}}\left\{\frac{-q+\frac{K_{w}}{\delta_{w}} \frac{\partial P_{c}}{\partial X} K-g K \sin \alpha\left(\frac{K_{0} \rho_{0}}{\delta_{0}}+\frac{K_{w} \rho_{w}}{\delta_{W}}\right)}{K \frac{K_{w}}{\delta_{w}}\left(1+\frac{K_{0}}{K_{w}} \frac{\delta_{w}}{\delta_{0}}\right)}-\frac{\partial P_{c}}{\partial X}+\rho_{w} g \sin \alpha\right\}\right] \\
& \frac{\partial}{\partial X}\left[\frac{K_{w} K}{\delta_{w}}\left\{\frac{-q+\frac{K_{w}}{\delta_{w}} \frac{\partial P_{c}}{\partial X} K-g K \sin \alpha\left(\frac{K_{0} \rho_{0}}{\delta_{0}}+\frac{K_{W} \rho_{W}}{\delta_{W}}\right)+\left(\frac{K_{W} K}{\delta_{W}}+\frac{K_{0} K}{\delta_{0}}\right)\left(\frac{-\partial P c}{\partial X}+\rho_{w} g \sin \alpha\right)}{K \frac{K_{w}}{\delta_{w}}\left(1+\frac{K_{0}}{K_{w}} \frac{\delta_{w}}{\delta_{0}}\right)}\right\}\right] \\
& \frac{\partial}{\partial X}\left[\frac{K_{w} K}{\delta_{w}}\left\{\frac{-q+\frac{K_{w}}{\delta_{w}} \frac{\partial P_{c}}{\partial X} K-g K \sin \alpha\left(\frac{K_{0} \rho_{0}}{\delta_{0}}+\frac{K_{W} \rho_{W}}{\delta_{W}}\right)+\left(\frac{K_{W} K}{\delta_{W}}+\frac{K_{0} K}{\delta_{0}}\right)\left(\frac{-\partial P c}{\partial X}+\rho_{w} g \sin \alpha\right)}{K \frac{K_{w}}{\delta_{w}}\left(1+\frac{K_{0}}{K_{w}} \frac{\delta_{w}}{\delta_{0}}\right)}\right\}\right] \\
& =\frac{\partial}{\partial X}\left[\frac{\frac{K_{W} K}{\delta_{W}}\left\{-q-\frac{K_{0}}{\delta_{0}} \frac{\partial P_{c}}{\partial X} K+g K \frac{K_{0}}{\delta_{0}}\left(\rho_{W}-\rho_{0}\right)\right\}}{K \frac{K_{W}}{\delta_{W}}\left(1+\frac{K_{0}}{K_{W}} \frac{\delta_{W}}{\delta_{0}}\right)}\right]
\end{aligned}
$$




\section{APPENDIX (B)}

$$
\begin{gathered}
\left(\frac{K_{W} K}{\delta_{W}}+\frac{K_{0} K}{\delta_{0}}\right) \frac{\partial P_{0}}{\delta_{w}} \frac{\partial P_{c}}{\partial X}+g K \sin \alpha\left(\frac{K_{0} \rho_{0}}{\delta_{0}}+\frac{K_{W} \rho_{W}}{\delta_{W}}\right)=-q \\
\frac{\partial P_{0}}{\partial X}=\frac{1}{2} \frac{\partial P_{c}}{\partial X}
\end{gathered}
$$

Substituting value of (B.2) in Eq. (B.1) we have

$$
\begin{gathered}
\left(\frac{K_{w}}{\delta_{w}}+\frac{K_{0}}{\delta_{0}}\right) \frac{1}{2} \frac{\partial P_{c}}{\partial X} K-\frac{K_{w}}{\delta_{w}} \frac{\partial P_{c}}{\partial X} K+g K \sin \alpha\left(\frac{K_{0} \rho_{0}}{\delta_{0}}+\frac{K_{w} \rho_{w}}{\delta_{w}}\right)+q=0 \\
\frac{1}{2} K\left(\frac{K_{0}}{\delta_{0}}-\frac{K_{w}}{\delta_{w}}\right) \frac{\partial P_{c}}{\partial X}+g K \sin \alpha\left(\frac{K_{w} \rho_{0}}{\delta_{0}}+\frac{K_{w} \rho_{w}}{\delta_{w}}\right)+q=0
\end{gathered}
$$

\section{APPENDIX (C)}

$$
P \frac{\partial S_{W}}{\partial t}+\frac{\partial}{\partial X}\left[\frac{\frac{K_{0} K}{\delta_{0}}\left\{\frac{\partial P_{c}}{\partial X}+g \sin \alpha\left(\rho_{0}-\rho_{w}\right)\right\}}{\left(1+\frac{K_{0} \delta_{W}}{K_{w} \delta_{0}}\right)}+\frac{q}{\left(1+\frac{K_{0} \delta_{W}}{K_{w} \delta_{0}}\right)}\right]=0
$$

Substituting the value of " $q$ " from Appendix (B).

\section{References}

Chouke, R.L. et. al. (1959). The instability of flow immiscible viscous liquid-liquid displacement in premeable media. Petrol Trans, AIME, p. 316.

Jecquard, P. and Sequier, P. (1940). Movement debeux fluids on contact unmilien porex. J. Mech. 1(4).

Scheidegger, A.E. and Johnson, E.F. (1961). The statistical behaviour of instabilities in displacement process in porous medium. Canadian Journal of Physic.

Scheidegger, A.E. (1960). The physics flow through process media. Univ. Torento Press, Torento, 217,224, 229, p. 229-231.

Verma, A.P. (1924). Statistical behavior of fingering in displacement process in heterogeneous porous medium with capillary pressure. Canadian Journal of Physic. 47(3), p. 319-324.

Table 1.

\begin{tabular}{|c|c|c|c|c|c|c|c|c|c|}
\hline$\eta$ & 0 & 0.1 & 0.2 & 0.3 & 0.4 & 0.5 & 0.6 & 0.7 & 0.8 \\
\hline$F(\eta)$ & 0.01 & 0.0064 & 0.0036 & 0.0016 & 0.0004 & 0.0 & 0.0004 & 0.0016 & 0.0036 \\
\hline
\end{tabular}

\begin{tabular}{|c|c|c|c|c|c|c|c|c|c|}
\hline$\eta$ & 0.9 & 1 & 1.1 & 1.2 & 1.3 & 1.4 & 1.5 & 1.6 & 1.7 \\
\hline$F(\eta)$ & 0.0064 & 0.01 & 0.0144 & 0.0196 & 0.0256 & 0.0324 & 0.04 & 0.0484 & 0.0576 \\
\hline
\end{tabular}

\begin{tabular}{|c|c|c|c|c|c|c|c|c|}
\hline$\eta$ & 1.8 & 1.9 & 2 & 2.1 & 2.2 & 2.3 & 2.4 & 2.5 \\
\hline$F(\eta)$ & 0.0676 & 0.0784 & 0.09 & 0.1024 & 0.1156 & 0.1296 & 0.1444 & 0.16 \\
\hline
\end{tabular}




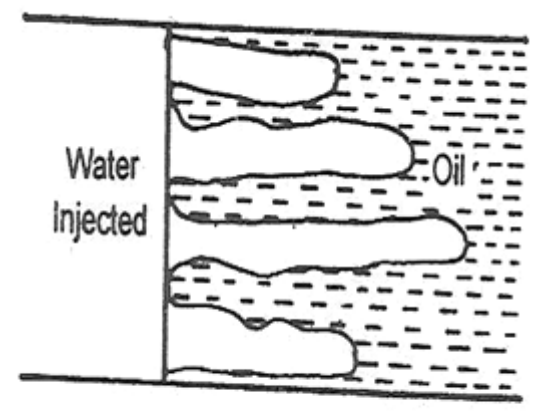

Fig.1(a)

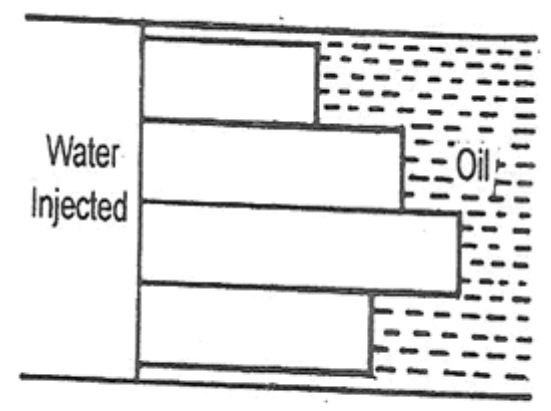

Fig. 1(b)

Figure 1.

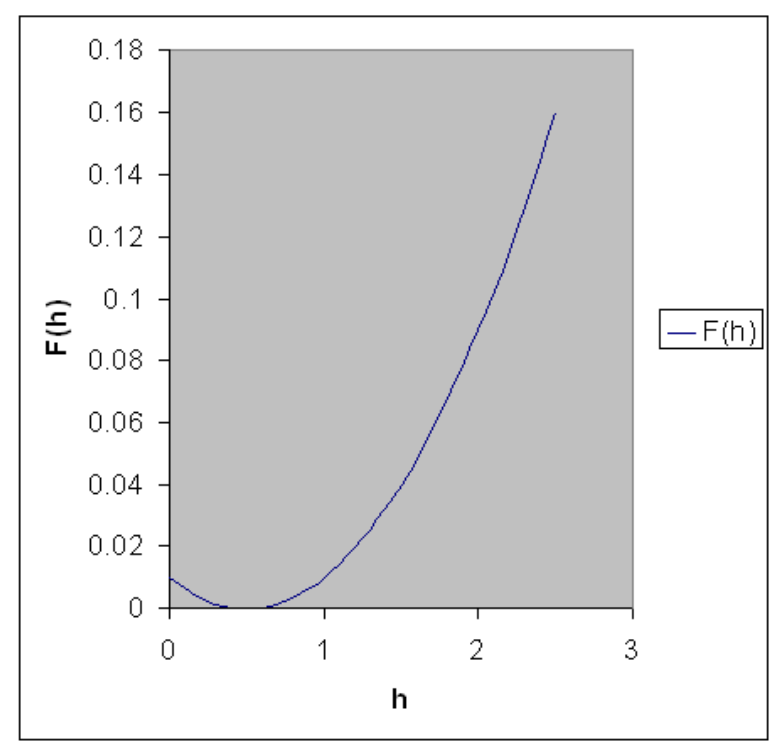

Figure 2. 